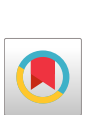

\title{
Misdiagnosis of Mycobacterium abscessus Pulmonary Infection as Pulmonary Tuberculosis
}

\author{
Sepehr Navid, ${ }^{1}$ and Masoud Keikha ${ }^{1,{ }^{*}}$ \\ ${ }^{1}$ Department of Microbiology, School of Medicine, Isfahan University of Medical Sciences, Isfahan, IR Iran \\ "Corresponding author: Masoud Keikha, Department of Microbiology, School of Medicine, Isfahan University of Medical Sciences, Isfahan, IR Iran. Tel: +98-9386836425, E-mail: \\ masoud.keykha90@gmail.com
}

Received 2018 February 28; Revised 2018 May 29; Accepted 2018 June 13.

\begin{abstract}
Introduction: Mycobacterium absessus is one of the important pathogens of rapidly growing mycobacteria, which can cause various infections in humans. Nontuberculosis pulmonary infection is similar to pulmonary tuberculosis and can be misdiagnosed as Mycobacterium tuberculosis. In this study, a case of pulmonary infection of M. abscessus is reported in healthy patients with previous pulmonary tuberculosis from Iran.

Case Presentation: The patient was a 85 year-old woman admitted to a hospital due to productive cough, dyspnea, fever, night sweats, weigh lost, hemoptysis, chest pain, anorexia, vomiting, and hematuria who had the history of pulmonary tuberculosis. According to clinical manifestation, laboratory examination, chest X-ray, and presence of acid-fast bacilli in direct smear, reactivation of tuberculosis was assumed and anti-TB chemotherapy was started; however, the patients' symptoms were not improved. The causative agent was isolated from the sputum samples of the patient and identified as Mycobacterium abscessus by using phenotypic tests and molecular method. According to the antibiotic susceptibility results, the patient was treated with linezolid, amikacin, and cefoxitin after two weeks.

Conclusions: This is the first report of pulmonary infection caused by M. abscessus in patients with a history of pulmonary tuberculosis in Iran. Therefore, for the purpose of patient management, final diagnosis and appropriate treatment, non-tuberculosis mycobacteria (NTM) should be identified to the species level.
\end{abstract}

Keywords: Tuberculosis, hsp65, Mycobacterium abscessus

\section{Introduction}

Non-tuberculosis mycobacteria (NTM) are ubiquitous environmental organisms, which were introduced as human pathogenic in 1990's. This group of bacteria can result in various infection including pulmonary, cutaneous, brain, eye, blood stream, and catheter-related infection in immune-compromised patients and even healthy individuals $(1,2)$.

According to the literature, several reports of drugresistant Mycobacterium tuberculosis (DR-TB) from developing countries are wrong. In fact, Mycobacterium spp. was not identified at species level in these laboratories, and anti-tuberculosis drugs were routinely recommend for these patients. However, given that non-tuberculosis mycobacteria (NTM) are resistant to anti-tuberculosis drugs; the patients were not cured and were misdiagnosed with DR-TB (3). According to the American thoracic society (ATS) guideline, identification and differentiation of clinical isolates of Mycobacterium spp. is essential for final diagnosis, appropriate treatment, patient management, clinical significance, and epidemiological studies $(1,4)$.

Mycobacterium abscessus was originally classified as subspecies of Mycobacterium chelonae by Moore and Frerichs in 1953; 23 later, this bacterium was reclassified as novel species by Kubica et al. Today, M. abscessus is consid- ered as a group of rapidly growing mycobacteria (RGM), which are emerged as an important pathogen in humans and recognized as the cause of approximately $65 \%-80 \%$ of lung disease induced by rapidly growing mycobacteria. $M$. abscessus is resistant to a wide range of antibiotics and its treatment is very difficult $(5,6)$. Here, we describe a case of M. abscessus pulmonary infection mimicking pulmonary tuberculosis in a healthy patient with a history of previous pulmonary TB.

\section{Case Presentation}

An 85-year-old woman admitted to the hospital due to productive cough, dyspnea, fever, night sweats, weigh lost, hemoptysis, chest pain, anorexia, vomiting, and hematuria is presented here. She had a history of previous pulmonary tuberculosis, however, no evidence for human immunodeficiency virus (HIV) infection or immune-disorder was observed. Chest X-ray showed lung diffuse infiltrates lesions (Figure 1), laboratory examination results were as follows: WBC 8,300 $\mu \mathrm{L}$, RBC $4.17 \mu \mathrm{L} \times 10^{6}$, platelet 297,000 $\mu \mathrm{L}$, Hemoglobin $10.9 \mathrm{~g} / \mathrm{dL}$ and Hematocrit 33.1\%; other laboratories indexes: BUN $24 \mathrm{mg} / \mathrm{dL}$, creatinine $0.9 \mathrm{mg} / \mathrm{dL}$, calcium $7.5 \mathrm{mg} / \mathrm{dL}$, phosphorus $3.4 \mathrm{mg} / \mathrm{dL}$, sodium $127 \mathrm{mEq} / \mathrm{L}$, potassium $2.2 \mathrm{mEq} / \mathrm{L}$, AST $26 \mathrm{U} / \mathrm{L}$, ALT $17 \mathrm{U} / \mathrm{L}$, CPK $55 \mathrm{U} / \mathrm{L}$, CPK-MB $9 \mathrm{U} / \mathrm{L}$, and troponin $\mathrm{I}<25 \mathrm{ng} / \mathrm{L}$. Urine culturing 
was negative and direct smear examination of sputum revealed the presence of acid-fast bacilli (AFB); based on the previous clinical history, reactivation of tuberculosis was assumed and anti-tuberculosis therapy with isoniazid, rifampin, and ethambutol was started. However, the patient did not improve and in the culture of sputum, mycobacterium spp. grew in Lowenstein-jensen and Sauton's agar (Figure 2). The isolate was identified using phenotypic tests consisting growth rate of $<7$ days, ability for growth on MacConkey agar without crystal violet, production of urease, positive for iron uptake, Aryl-sulfatase (3 days), heat stable catalase, hydrolysis of tween 80 and negative results for pigment, niacin production, and nitrate reduction. Following DNA extraction (simple boiling method), amplification and sequencing of the nearly full length of 16S rRNA gene and partial segment of hsp65 gene was carried out for this isolate as previously described (1). The hsp65 gene sequence of the isolate showed $100 \%$ similarities with the corresponding sequence of mycobacterium abscessus strain ATCC 19977. The GenBank accession number of this isolate is MG930482.

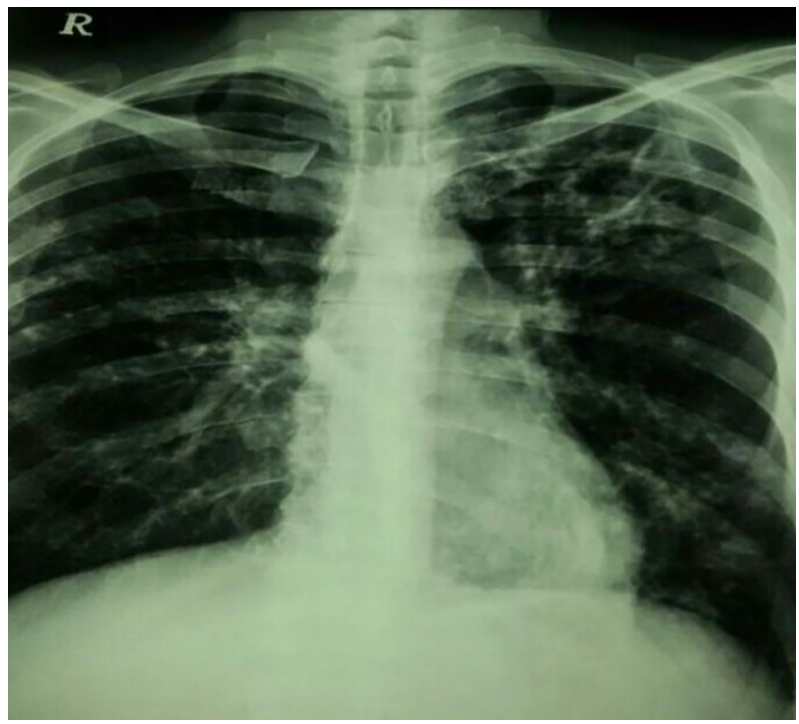

Figure 1. Chest X-ray of the patient

The antibiotic susceptibility testing was performed using micro-broth dilution as recommended by clinical and laboratory standards institute (CLSI) (7). The antibiotic sensitivity test showed that the isolate was sensitive to amikacin, cefoxitin, and linezolid. Moreover, this isolate exhibited resistance to clarithromycin and doxycycline. Finally, the patient was treated with linezolid (600 mg twice a day), amikacin (15 mg/kg/d), and cefoxitin (200 mg/kg/d) for six months. The patient was completely recovered after

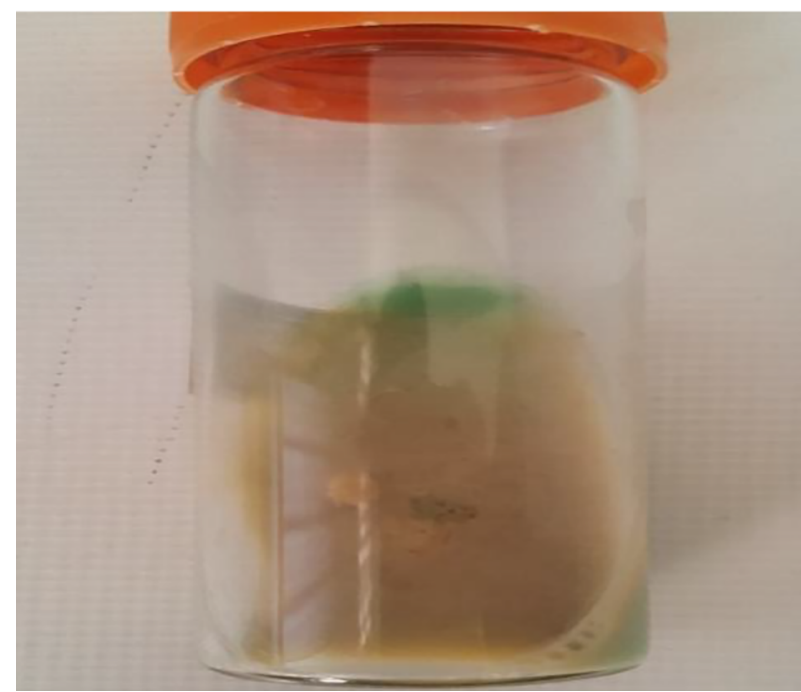

Figure 2. Mycobacterium abscessus growth on Lowenstein- jensen

two weeks.

\subsection{Ethical Statement}

Formal consent for publication was obtained from the patient.

\section{Discussion}

Given the lack of expert technicians in TB endemic developing countries, mycobacterial infection could be diagnosed as $M$. tuberculosis based on the presence of acid fast bacilli in direct smear microbiology. Consequently, the patients would be treated with anti-tuberculosis drugs and regarding no response to TB-drugs, the disease may be presumed as drug-resistant TB (3). Identification and differentiation of NTM using phenotypic test are difficult to standardize, in addition to its high cost, need to expertise staff, being time-consuming, labor-intensive; it may be even wrong in some cases. However, molecular methods such as PCR-restriction fragment length polymorphism (PCR-RFP) and sequencing using housekeeping genes (for example16S rRNA,16S-23S rRNA internal transcribed spacer (ITS), rpoB, $h s p 65, r e c A, \ldots)$ are inexpensive, rapid, accurate, and reliable for identification of NTM to species level (1).

M. abscessus was first identified by Moore and Frerichs from an infection of the knee with subcutaneous abscesslike lesions as subspecies of M. chelonae. However, according to the studies of Kusunoki and Ezaki, it was determined as novel distinct species as M. abscessus. Nowadays, the taxonomic scientists declared that Mycobacterium abscessus comprise three closely related subspecies of $M$. abscessus 
subsp. abscessus, M. abscessus subsp. Massiliense, and M. abscessus subsp. bolletii. All members of M. ascessus complex were isolated from clinical specimens, particularly lung disease $(6,8)$. According to the literature, isolation rate of M. abscessus and M. massiliense are approximately equal, however, $M$. bolleti is rare; clinical manifestation of $M$. $a b$ scessus complex are similar, nonetheless, antibiotic resistance (particularly, clarithromycin) in M. abscessus subsp. abscessus is more common $(6,9)$.

M. abscessus is one of the important groups of RGM (Rapidly growing mycobacteria), which are able to cause serious infection in immune-compromised patients as well as healthy people. This group of mycobacteria can contaminate medical instruments and cause disseminated infection in patients who use catheters and other medical equipment $(2,10)$. One of the main points about M. abscessus-caused human infection is its antibiotic resistance. Many NTM species are susceptible to new macrolides and can be treated with clarithromycin and azithromycin. However, unfortunately, M. abscessus infections are inefficient to macrolide-based chemotherapy due to overexpression of erm (erythromycin ribosomal methylase gene) or production of biofilm (9-12). Combinational therapy of amikacin with cefoxitin or imipenem and oral macrolides has been recommended by American thoracic society and infectious diseases society of America. However, insufficient treatment response rates of M. abscessus infections, optimal therapeutic regimens, and treatment durations are not well established and challengeable (46). Paradoxically, there has been evidence for person-toperson transmission of M. abscessus between patients with cystic fibrosis $(12,13)$.

In conclusion, M. abscessus can cause pulmonary infection mimicking pulmonary tuberculosis. The present study emphasized the problems about identification of NTM in developing countries, and highlighted misdiagnosis of NTM pulmonary infection with tuberculosis. Precise identification should be determined based on the standard microbiologic protocols, however, previous history of patients and clinical manifestation are not completely reliable. Furthermore, molecular methods such as sequencing of the 16S rRNA, rpoB, and hsp65 can help us in rapid (in urgent cases), reliable, and efficient identification of NTM at the species level.

\section{Footnote}

Conflicts of Interests: The authors declare that there are no conflicts of interest.

\section{References}

1. Hashemi-Shahraki A, Bostanabad SZ, Heidarieh P, Titov LP, Khosravi $\mathrm{AD}$, Sheikhi N, et al. Species spectrum of nontuberculous mycobacteria isolated from suspected tuberculosis patients, identification by multi locus sequence analysis. Infect Genet Evol. 2013;20:312-24. doi: 10.1016/j.meegid.2013.08.027. [PubMed: 24070831].

2. Wagner D, Young LS. Nontuberculous mycobacterial infections: a clinical review. Infection. 2004;32(5):257-70. [PubMed: 15624889].

3. Shahraki Hashemi A, Heidarieh P, Zaker Bostanabad S, Khosravi $\mathrm{AD}$, Hashemzadeh M, Khandan S, et al. "Multidrug-resistant tuberculosis" may be nontuberculous mycobacteria. Eur J Intern Med. 2015;26(4):279-84. doi: 10.1016/j.ejim.2015.03.001.

4. Griffith DE, Aksamit T, Brown-Elliott BA, Catanzaro A, Daley C, Gordin F, et al. An official ATS/IDSA statement: diagnosis, treatment, and prevention of nontuberculous mycobacterial diseases. Am J Respir Crit Care Med. 2007;175(4):367-416. doi: 10.1164/rccm.200604-571ST. [PubMed: 17277290].

5. Tortoli E, Kohl TA, Brown-Elliott BA, Trovato A, Leao SC, Garcia MJ, et al. Emended description of Mycobacterium abscessus, Mycobacterium abscessus subsp. abscessus and Mycobacteriumabscessus subsp. bolletii and designation of Mycobacteriumabscessus subsp. massiliense comb. nov. Int J Syst Evol Microbiol. 2016;66(11):4471-9. doi: 10.1099/ijsem.0.001376. [PubMed: 27499141].

6. Koh WJ, Jeon K, Lee NY, Kim BJ, Kook YH, Lee SH, et al. Clinical significance of differentiation of Mycobacterium massiliense from Mycobacterium abscessus. Am J Respir Crit Care Med. 2011;183(3):405-10. doi: 10.1164/rccm.201003-03950C. [PubMed: 20833823].

7. Hawkins C, Qi C, Warren J, Stosor V. Catheter-related bloodstream infections caused by rapidly growing nontuberculous mycobacteria: a case series including rare species. Diagn Microbiol Infect Dis. 2008;61(2):187-91. doi: 10.1016/j.diagmicrobio.2008.01.004. [PubMed: 18294801]

8. Kusunoki S, Ezaki T. Proposal of Mycobacterium peregrinum sp. nov., nom. rev., and elevation of Mycobacterium chelonae subsp. abscessus (Kubica et al.) to species status: Mycobacterium abscessus comb. nov. Int J Syst Bacteriol. 1992;42(2):240-5. doi: 10.1099/00207713-42-2240. [PubMed: 1581184].

9. Nash KA, Brown-Elliott BA, Wallace RJ. A novel gene, erm(41), confers inducible macrolide resistance to clinical isolates of Mycobacterium abscessus but is absent from Mycobacterium chelonae. Antimicrob Agents Chemother. 2009;53(4):1367-76. doi: 10.1128/AAC.0127508. [PubMed: 19171799]. [PubMed Central: PMC2663066].

10. Howard ST, Byrd TF, Lyons CR. A polymorphic region in Mycobacterium abscessus contains a novel insertion sequence element. Microbiology. 2002;148(Pt 10):2987-96. doi: 10.1099/00221287-148-10-2987. [PubMed: 12368432].

11. Nessar R, Cambau E, Reyrat JM, Murray A, Gicquel B. Mycobacterium abscessus: a new antibiotic nightmare. J Antimicrob Chemother. 2012;67(4):810-8. doi: 10.1093/jac/dkr578. [PubMed: 22290346].

12. Bryant JM, Grogono DM, Greaves D, Foweraker J, Roddick I, Inns $\mathrm{T}$, et al. Whole-genome sequencing to identify transmission of Mycobacterium abscessus between patients with cystic fibrosis: a retrospective cohort study. Lancet. 2013;381(9877):1551-60. doi: 10.1016/S0140-6736(13)60632-7. [PubMed: 23541540]. [PubMed Central: PMC3664974].

13. Jonsson BE, Gilljam M, Lindblad A, Ridell M, Wold AE, WelinderOlsson C. Molecular epidemiology of Mycobacterium abscessus, with focus on cystic fibrosis. J Clin Microbiol. 2007;45(5):1497-504. doi: 10.1128/JCM.02592-06. [PubMed: 17376883]. [PubMed Central: PMC1865885] 